\title{
Système semencier et pureté des semences dans un contexte d'utilisation d'OGM : le cas du coton Bt au Burkina Faso
}

\author{
Larbouga Bourgou ${ }^{1}$ et Michel Fok ${ }^{2,3, *}$ \\ 1 INERA, Programme Coton, BP 208, Bobo-Dioulasso, Burkina Faso \\ 2 CIRAD, UPR AIDA, F-34398 Montpellier, France \\ 3 AIDA, Univ. Montpellier, CIRAD, Montpellier, France
}

\begin{abstract}
Résumé - Depuis la commercialisation du coton $\mathrm{Bt}$, la question de la pureté des semences utilisées par les producteurs est peu abordée, encore moins en relation avec l'analyse du système semencier dans le pays concerné. Cet article étudie le cas du Burkina Faso en 2015, pays où la couverture des surfaces en coton transgénique avec deux gènes Bt est restée partielle, jusqu'à sa suspension décidée la même année. En se basant sur les seuils de présence de gènes Bt retenus dans l'étude pour déclarer la nature conventionnelle ou transgénique des parcelles échantillonnées, $29,3 \%$ des parcelles supposées conventionnelles l'étaient effectivement, alors que $92,6 \%$ des parcelles supposées transgéniques l'étaient effectivement. Par contre, seulement $40 \%$ des parcelles supposées transgéniques l'étaient bien si l'on se base sur le seuil de présence de gènes $\mathrm{Bt}$ attendu dans des parcelles transgéniques effectivement installées avec des semences Bollgard II suffisamment pures. L'examen du système semencier permet de lui attribuer une partie des défauts de pureté observés. Par ailleurs, si l'on considère la chronologie des événements jusqu'à la livraison des semences transgéniques par Monsanto, ces semences ne pouvaient pas contenir systématiquement les deux gènes Bt. Un tel défaut initial s'est maintenu, voire accentué par la suite. Il a certainement réduit dès le départ l'efficacité du coton Bt et, pis encore, la durabilité de son utilisation en facilitant la sélection de ravageurs résistants à chacun des deux gènes utilisés, voire simultanément aux deux. Le cas étudié souligne l'importance, pour l'efficacité et la durabilité de l'utilisation des semences transgéniques, d'avoir un système semencier qui assure et préserve la pureté des semences produites. Il révèle aussi, indépendamment de l'utilisation de ces semences, le besoin d'ajuster le système internalisé de production de semences cotonnières en Afrique francophone.
\end{abstract}

Mots clés : coton conventionnel / OGM / dispositif de production de semences / contrôle des semences

Abstract - Seed production scheme and purity under GMO use: the case of Bt-cotton in Burkina Faso. Since the commercial release of Bt cotton, the issue of the purity of the seeds used by producers has been little addressed, further less in relation to the analysis of the seed production and distribution system in the country concerned. This paper examines the case of Burkina Faso in 2015, a country where transgenic cotton with two Bt genes was planted alongside conventional cotton until its suspension decided the same year. According to the thresholds for the presence of Bt genes retained in the study to declare the conventional or transgenic nature of the sampled plots, only $29.3 \%$ of the plots assumed to be conventional were indeed conventional, compared to $92.6 \%$ of the supposedly transgenic plots which were indeed transgenic. However, among transgenic plots, only $40 \%$ met the threshold for containing both of the Bt cotton genes that would have been expected if they had been planted with pure Bollgard II seeds. Some of these purity defects may be explained by examining the system of seed production and distribution. In addition, if one considers the chronology of events up to the delivery of the transgenic seeds by Monsanto, these seeds could not systematically contain the two Bt genes. Such an initial defect was maintained or even accentuated afterwards. It hampered the efficacy of Bt cotton from the outset and, worse, the sustainability of its use by facilitating the selection of pests resistant to each of the two genes used, or even to both simultaneously. The case study underlines how important it is, in view of an efficient and sustainable use of transgenic seeds, to produce and preserve seed purity. It also reveals, regardless of the use of transgenic seeds, the need to adjust the internalized scheme of cotton seed production in francophone Africa.

Keywords: conventional cotton / GMO / seed production scheme / seed control

\footnotetext{
$\overline{\text { *Auteur de correspondance }}$ : michel.fok@cirad.fr
} 


\section{Introduction}

Depuis la commercialisation du coton génétiquement modifié, en particulier du coton $\mathrm{Bt}$ rendu résistant à certains ravageurs, la question de la pureté des semences a été peu abordée et ne l'a été que du point de vue de la culture du coton conventionnel. Les études soulignant la perte de pureté des semences conventionnelles sont liées à la préoccupation des producteurs de coton biologique de satisfaire à l'exigence d'absence d'organismes génétiquement modifiés (OGM) au seuil imposé (Cederholm, 2014). Aux États-Unis, la coexistence de cultures conventionnelles et transgéniques est perçue comme une illusion, tant la contamination par les cultures transgéniques est jugée inévitable (Food Democracy Now!, 2014), rendant impossible la poursuite de la production de coton biologique.

Les études publiées sur la pureté des semences transgéniques, plus précisément celles du coton $\mathrm{Bt}$, sont rares. De nombreux travaux de recherche ont porté sur l'expression des gènes Bt dans les cotonniers, en mesurant la concentration des toxines Bt, comme par exemple l'effet des descendants F2 des hybrides F1 que des agriculteurs indiens pouvaient utiliser (Singh et al., 2016). La question de la pureté des semences de coton Bt n'a été spécifiquement abordée qu'au Pakistan, par l'analyse en laboratoire d'échantillons de plantes prélevés dans les champs des paysans (Spielman et al., 2017). Même dans les manuels techniques officiels pour la coexistence des deux types de coton, la préservation de la pureté du coton conventionnel est l'objet de recommandations, mais pas le taux de pureté des semences transgéniques (USDA, 2015), comme s'il n'y avait pas de problème à ce sujet.

Les études sur l'utilisation du coton Bt en relation avec les conditions de production et de distribution des semences sont encore plus rares, limitées surtout au cas de la Chine. Fok et Xu (2010) ont analysé conjointement le développement du marché des semences en relation avec celui des variétés de coton Bt en Chine, développement conjoint considéré comme facteur et contrainte de l'adoption de ce coton dans le pays (Fok et $\mathrm{Xu}$, 2011). Les caractéristiques et fonctionnements des systèmes semenciers ont été occultés en général dans l'évaluation des expériences d'utilisation des OGM en agriculture, hormis les craintes sur la dépendance semencière.

Cet article analyse la pureté réelle des semences en situation d'utilisation de coton Bt au Burkina Faso, au regard de la présence des gènes $\mathrm{Bt}$ dans les semences conventionnelles ou transgéniques, en relation avec les modalités d'introduction du coton $\mathrm{Bt}$ et le système semencier du pays. Jusqu'à la suspension en 2016 du coton Bt vulgarisé en 2008 (Bollgard II, avec les deux gènes Bt Cry1Ac et Cry2Ab), ce coton a cohabité avec le coton conventionnel.

\section{Coton Bt et dispositifs semenciers}

\subsection{Introduction, adoption et distribution du coton Bt au Burkina Faso}

L'adoption du coton Bt au Burkina Faso a résulté d'un processus de près de quinze ans et remontant à 1992 quand le pays avait pris part aux négociations sur la Convention de la diversité biologique, aboutissant au Protocole de Cartagena qu'il a ratifié dès 2003 (Zangré, 2009). C'est en 1998 que les représentants du Burkina Faso ont rencontré Monsanto pour la première fois, rencontre qui aboutira à la vulgarisation du coton Bt dix ans plus tard, dont cinq ans d'expérimentation sur l'efficacité et les impacts environnementaux.

Dès le départ, le Burkina Faso avait retenu les options de recourir à la transformation des variétés qu'il utilisait et à la production locale des semences. Trois variétés ont été fournies en 2004 pour transformation, mais celle-ci a été menée à son terme seulement pour deux d'entre elles ( $c f$. infra). Dans l'accord établi avec Monsanto, il était retenu d'intégrer la production des semences de coton $\mathrm{Bt}$ dans le système de production de semences existant au Burkina Faso, en impliquant l'Institut de l'environnement et de recherches agricoles du Burkina Faso (INERA) et la Société burkinabé des fibres textiles (SOFITEX), qui avaient une grande expérience en la matière (Zangré, 2009). Le contrôle de la qualité de la semence de coton BG II devait être rigoureusement effectué, pour notamment s'assurer de la présence du gène $\mathrm{Bt}$ dans au moins $98 \%$ des semences, avec un taux de confiance statistique d'au moins $95 \%$ (AICB, 2015). Il n'est indiqué, dans aucun document dont nous avons connaissance, les modalités pour parvenir au niveau de qualité mentionnée à partir des semences que Monsanto allait fournir.

L'accord signé stipule que Monsanto devait fournir au Burkina Faso 275 tonnes de semences G2 (ou semences de prébase de grande pureté génétique, deuxième génération de descendance des semences G0 correspondant au matériel que le sélectionneur doit reproduire à l'identique chaque année), réparties entre la variété FK95 BG II et FK96 BG II (versions $\mathrm{Bt}$ respectivement des variétés FK37 et Stam 59A). Ces semences ont été livrées en juin 2008 et ont toutes servi à la multiplication des semences certifiées au cours de la campagne 2008-2009 (AICB, 2015). Nous n'avons trouvé nulle mention d'actions pour entretenir ou constituer les semences de base ou de pré-base.

Le défaut de pureté génétique des semences livrées en juin 2008 ressort de la chronologie restituée ci-dessus. La durée entre la fourniture des variétés à transformer et la livraison des semences « $\mathrm{G} 2$ » a permis de réaliser deux back-crosses après le croisement avec une variété américaine "donneuse» de gènes Bt. Le manque de pureté concerne un insuffisant retour vers le génotype des variétés africaines, induisant un défaut de longueur de fibre dont Fok (2016) a estimé l'impact financier. Le manque de pureté concerne aussi, et peut-être davantage encore, la présence effective et simultanée des deux gènes Bt. Techniquement, il était impossible que toutes les semences fournies contiennent des gènes $\mathrm{Bt}$, encore moins de manière simultanée. Le processus de back-cross faisait croiser plusieurs fois de suite avec les variétés africaines fournies, aussi la présence des gènes Bt pouvait-elle se diluer si aucune disposition n'était prise, ou pas suffisamment, pour s'assurer de la présence des gènes $\mathrm{Bt}$ à la descendance de chaque backcross. Aucun document, à notre connaissance, n'informe sur de telles dispositions.

Le manque de pureté analysé ci-dessus indique que la dénomination G2 des semences fournies en 2008 était inappropriée, abusive et trompeuse. Le caractère trompeur a le plus d'incidence négative car il a pu faire croire à une grande pureté génétique et à ne pas engager de travail de sélection au niveau de la recherche burkinabé pour parfaire la pureté des semences. 


\subsection{Dispositifs de production et de distribution de semences}

Les dispositifs de production et de distribution de semences cotonnières en Afrique francophone, et au Burkina Faso en particulier, ont évolué mais ont gardé une forte empreinte d'internalisation au sein des sociétés cotonnières, laissée par la Compagnie française de développement des textiles (CFDT). Fok (1993) a rappelé que la CFDT, en succédant à l'Union cotonnière de l'Empire français, avait d'abord repris l'activité principale de cette dernière, qui était d'expérimenter et de distribuer des semences aux producteurs (Rabault, 1944).

C'est à partir de 1954 que, forte de pouvoir également commercialiser le coton-graine, la CFDT mit en œuvre son approche de villages semenciers pour y concentrer ses actions d'appui et de contrôle, de commercialisation des productions, d'évacuation et de suivi de l'égrenage dans des unités dédiées. Même si ce système a évolué par la suite ( $c f$. infra), deux de ses caractéristiques ont demeuré. Il s'agit du traçage jusqu'au village ou au producteur, mais pas jusqu'à la parcelle. Par ailleurs, il n'y avait pas de prix différencié à l'achat des productions destinées aux semences, même si certaines compensations pouvaient être appliquées par la suite en réponse à une plus grande utilisation d'intrants (cas actuel du Burkina Faso).

Le modèle de villages semenciers a opéré avec succès dans tous les pays cotonniers d'Afrique jusque vers la fin des années 1980, avec des niveaux de production bien plus faibles et peu de différenciation entre les producteurs. Les séquences de changement variétal en témoignent. Fok (1995) a montré qu'au cours de la période 1955-1995, le nombre de variétés vulgarisées dans les neuf pays cotonniers de l'Afrique francophone (Sénégal, Mali, Burkina Faso, Bénin, Togo, Côte d'Ivoire, Tchad, Cameroun et République centrafricaine) a varié de 7 à 15 (14 au Burkina Faso), soit une durée moyenne de vie d'une variété de 4,4 ans. Les productions nationales des pays concernés étaient cependant seulement de 100 à 150 milliers de tonnes de coton-graine (contre sept fois plus au Bénin et au Mali aujourd'hui) et les plus gros producteurs cultivaient quelques hectares de coton contre quelques dizaines maintenant.

Le processus associatif au sein des villages dans les zones cotonnières, puis l'émergence de «gros producteurs» de coton, ont fait évoluer l'approche initiale de villages semenciers. Le développement du processus a engendré assez rapidement l'établissement de groupements multiples de producteurs cotonniers dans un même village (Fok, 1993), donnant lieu à une approche de groupements semenciers dans laquelle tous les groupements d'un village pouvaient rarement être impliqués tous à la fois. Il en découle une cohabitation de parcelles semencières et non-semencières dans les villages, accroissant la contrainte d'isolement à gérer. Il en découle aussi une cohabitation de deux types de production, semencière et non-semencière, alors que les transferts de production entre les paysans ont été avérés (l'explicitation des raisons nous ferait sortir du cadre de cette étude). Ces pratiques ont pu induire la contamination des productions destinées aux semences si des paysans semenciers y étaient impliqués.

L'approche de groupements semenciers a été complétée, voire suppléée, par celle des paysans semenciers, comme au
Burkina Faso où ces derniers sont considérés en priorité dans le programme semencier (Savadogo, 2015). C'est le résultat de l'émergence de gros producteurs de coton, dont la production permet de remplir un camion à l'évacuation vers l'usine d'égrenage, donnant lieu aux «achats directs » auprès de ces producteurs. Il en découle la cohabitation possible dans un même village de groupements semenciers, en fait une partie des groupements présents, et de paysans semenciers, accentuant les contraintes d'isolement des parcelles et les risques de mélange dans les productions si le respect de l'isolement n'est pas contrôlé correctement.

Le risque de mélange est accentué au Burkina Faso du fait de plusieurs facteurs, liés d'une part à l'utilisation dès 2005 de semences délintées conjointement avec les semences vêtues, et d'autre part, à l'attachement des paysans à semer à forte dose. La qualité germinative mieux assurée des semences délintées a conduit à réduire, à l'instar de tous les pays ayant adopté ces semences, les doses distribuées à l'hectare (d'abord $8 \mathrm{~kg} / \mathrm{ha}$ à la première campagne en 2008 , contre $30 \mathrm{~kg} /$ ha pour les semences vêtues). Les récriminations des producteurs contre la faible dose de semences délintées distribuée à l'hectare, car contraire à leur pratique de semis à forte dose sur un sol peu humide en début de saison, ont amené la Sofitex à augmenter les doses de semences délintées à $15 \mathrm{~kg} / \mathrm{ha}$. Pour certains paysans, cette augmentation restait insuffisante, mais à d'autres agriculteurs, elle donnait la latitude de semer une superficie supérieure à ce qui était prévu ou de donner des semences à d'autres agriculteurs. Par ailleurs, l'avènement du coton Bt ayant amené à délinter prioritairement les semences de ce type de coton (pour la campagne 2013-2014 par exemple, 94,2\% des semences délintées étaient $\mathrm{Bt}$ ), il en a découlé que les paysans fournis avec des semences délintées de coton $\mathrm{Bt}$ pouvaient alors ressentir davantage le besoin de compléter les semences obtenues, et ils pouvaient s'en procurer plus facilement auprès des utilisateurs de semences vêtues, plus fréquemment utilisateurs de coton conventionnel. Enfin, l'option de la Sofitex de surproduire en semences, pour s'affranchir de tout risque d'en manquer en cas d'un fort besoin de resemis pour raison climatique, est un autre facteur de mélange dans la production de semences. Sur une période de trois campagnes, Savadogo (2015) a calculé que les quantités de semences produites ou distribuées auraient permis de réaliser respectivement 3,00 et 1,67 fois les superficies finalement ensemencées, ce qui était bien supérieur à la norme de 1,25 que retenait déjà la Sofitex sur la base d'une dose élevée à l'hectare. En rendant plus facile pour les paysans d'acquérir les semences au-delà du plan initial d'emblavement, l'option de surproduction de semences accroît les risques de contrôle défaillant si les moyens ne sont pas adaptés à la dimension du programme semencier.

De manière étonnante, il n'existe pas, ou pas encore, de documents de référence décrivant les procédures à suivre pour la production de semences cotonnières au sein des sociétés ou des filières cotonnières. À notre connaissance, le Burkina Faso est le seul pays, depuis fin 2019, engagé dans la voie d'élaboration d'un tel document dans l'optique d'une certification des semences par un service national. Le travail de Savadogo (2015) au Burkina Faso est le seul donnant une vision d'ensemble de l'organisation pour la production et la distribution des semences au sein d'une société cotonnière. $\mathrm{Ce}$ travail met en évidence qu'en absence d'un document de 
procédures, ce sont les notes de service (parfois actualisées) de la Sofitex qui spécifiaient les règles à respecter à toutes les étapes où la pureté des semences produites pouvait être affectée négativement, de l'identification des zones et des paysans semenciers jusqu'à la distribution des semences aux paysans, après les opérations de commercialisation, de transport et d'égrenage pour obtenir les graines destinées aux semences, puis de délintage éventuel des semences et de leur mise en sac. Ce sont tous ces niveaux qui sont actuellement considérés dans la rédaction du document mentionné plus haut. L'approche de production internalisée se traduit par l'opération d'un « Service semence » au sein de la Sofitex, chargé de veiller aux bonnes pratiques regroupées dans les actions suivantes:

- établir le programme semencier pluriannuel et les plans semenciers annuels;

- organiser, suivre, contrôler les multiplications semencières;

- planifier les évacuations des productions destinées aux semences ;

- superviser les opérations à l'usine et au contrôle de pureté et de qualité des semences;

- suivre la mise en place des semences dans les villages.

Le Service semence est relayé dans les sept régions cotonnières par la section de suivi-évaluation, qui a des activités diverses de suivi au-delà de la question semencière. Cette section est elle-même relayée auprès des paysans par des agents de suivi de diverses activités. C'est notamment à ces agents qu'incombent le choix des paysans et/ou des groupements semenciers et la responsabilité de faire respecter les distances d'isolement et de prendre les dispositions pour prévenir les mélanges dans les productions destinées aux semences.

L'adoption du coton Bt n'a pas apporté de modification notable aux activités semencières au sein de la Sofitex ou aux modalités de leur exécution. Le seul changement a concerné le contrôle de la qualité transgénique des semences via des tests enzymatiques sur des échantillons de graines (Sofitex, s.d.). Le nombre de ces tests est respectivement de 11,22, 44 et 88 pour des champs de 1 à 5,5 hectares (ou lots de moins de 4,8 tonnes), des champs de 5,5 à 10,5 ha (ou lots de 4,8 à 10,5 tonnes), des champs de 10,5 à 25,5 ha (ou lots de 10,6 à 25,5 tonnes) et des champs de plus de 25,5 ha (ou lots de plus de 25,5 tonnes).

Le travail de Savadogo (2015) permet de disposer d'un regard critique sur la conduite des opérations semencières par une société cotonnière. Ce travail, basé sur des observations aux différents maillons de la chaîne de production de semences et sur des entretiens pour partager ces observations avec les agents de la Sofitex aux divers niveaux hiérarchiques, nuance très fortement le sentiment de qualité générale d'exécution qu'on pouvait avoir. Ces agents ont corroboré les manquements constatés à tous les maillons de la chaîne, qu'il serait trop long de restituer ici. Il en ressort que les mélanges de production lors $\mathrm{du}$ processus de production semencière pouvaient intervenir à tous les stades, des champs jusqu'aux usines d'égrenage et lors de toutes les phases de transfert. Dans les champs par exemple, les zones, les paysans ou les groupements pour la production semencière, n'étaient pas sélectionnées sur la base de critères clairement établis et les parcelles semencières n'étaient pas suivies, ou pas systématiquement, et le plus souvent tardivement. Quant au niveau des usines d'égrenage, le respect des règles de nettoyage des égreneuses était très discutable.

En raison des manquements évoqués ci-dessus dans l'exécution des diverses opérations par la Sofitex, l'amélioration du système de production de semences cotonnières (visée dans la rédaction mentionnée de procédures) doit tenir compte des pratiques des paysans dans l'utilisation des semences reçues, qui peuvent accentuer les risques de mélange dans la production de semences. Pour satisfaire le besoin d'un complément de semences, pour resemer ou pour semer plus que prévu, les paysans peuvent préférer s'adresser à leurs pairs, et non à la Sofitex. Cette préférence s'est accentuée avec la vulgarisation du coton $\mathrm{Bt}$, qui a rendu plus strictes les règles ou l'application des règles de distribution des semences, notamment celles du coton Bt (Luna et Dowd-Uribe, 2020). Dans ces arrangements entre paysans, qui peuvent concerner aussi les paysans impliqués dans la production de semences, on peut craindre que le souci sur les quantités à obtenir prime sur celui de la nature et de la variété des semences.

\section{Matériels et méthodes sur la pureté des semences}

Les données utilisées sont les résultats de tests ELISA pour attester d'une part de la vraie nature conventionnelle ou transgénique des parcelles échantillonnées et d'autre part du degré de pureté des semences utilisées pour l'installation de ces parcelles. Cela a été exécuté pour cerner la présence ou le degré de présence des gènes $\mathrm{Bt}$ (Cry1 Ac et Cry $2 \mathrm{Ab}$ ) dans les échantillons prélevés dans les parcelles des paysans. Les données ont été recueillies en décembre 2015 dans la zone d'intervention de la Sofitex, dans le cadre d'une étude visant à déterminer l'écart de qualité de la fibre entre les variétés de coton conventionnel et $\mathrm{Bt}$ cultivées à cette période (Bourgou et al., 2020).

En raison de l'organisation en place avec la Sofitex comme source exclusive des semences aux paysans, la nature des champs à échantillonner pouvait être facilement connue à partir des documents tenus par les «agents techniques coton» sur le terrain. L'information obtenue pouvait être considérée de prime abord comme fiable pour réaliser l'échantillonnage des champs selon leur nature conventionnelle ou transgénique. Pour autant, compte tenu des pratiques évoquées d'arrangements entre les paysans, le degré de fiabilité nécessite d'être cerné a posteriori.

Pour l'étude réalisée, l'échantillon des champs a couvert tous les districts de la zone d'intervention de la Sofitex (Fig. 1). Trois groupements de producteurs de coton (GPC) ont été sélectionnés au hasard par district, puis trois producteurs par GPC. Au sein des GPC, les champs de producteurs cultivant les deux types de coton ont été échantillonnés de préférence, complétés au besoin par des producteurs cultivant l'un des deux types. Le tableau 1 récapitule la situation d'échantillonnage avec une plus grande part de champs en coton $\mathrm{Bt}$, de manière conforme à la part de $62,53 \%$ que ce type de coton avait représenté dans la superficie totale semée en 2015.

Dans chaque champ, un échantillon d'un kilogramme de coton-graine a été prélevé au hasard sur 135 plants de coton en 


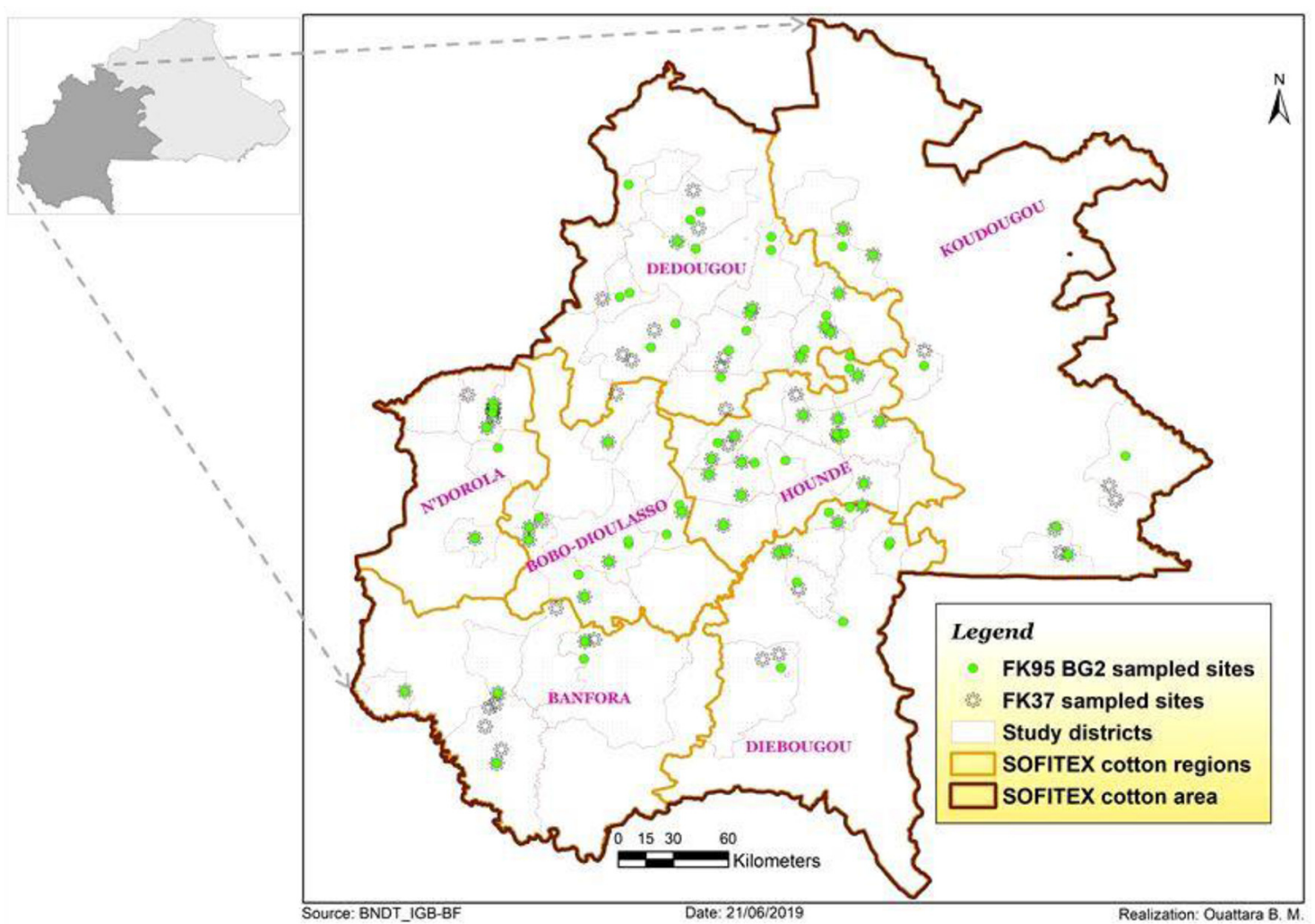

Fig. 1. Zone cotonnière de la Sofitex, montrant les villages et les sites échantillonnés.

Fig. 1. Sofitex cotton zone, showing study sampled districts and sites.

Tableau 1. Répartition des parcelles échantillonnées de l'étude.

Table 1. Distribution of the study sampled plots.

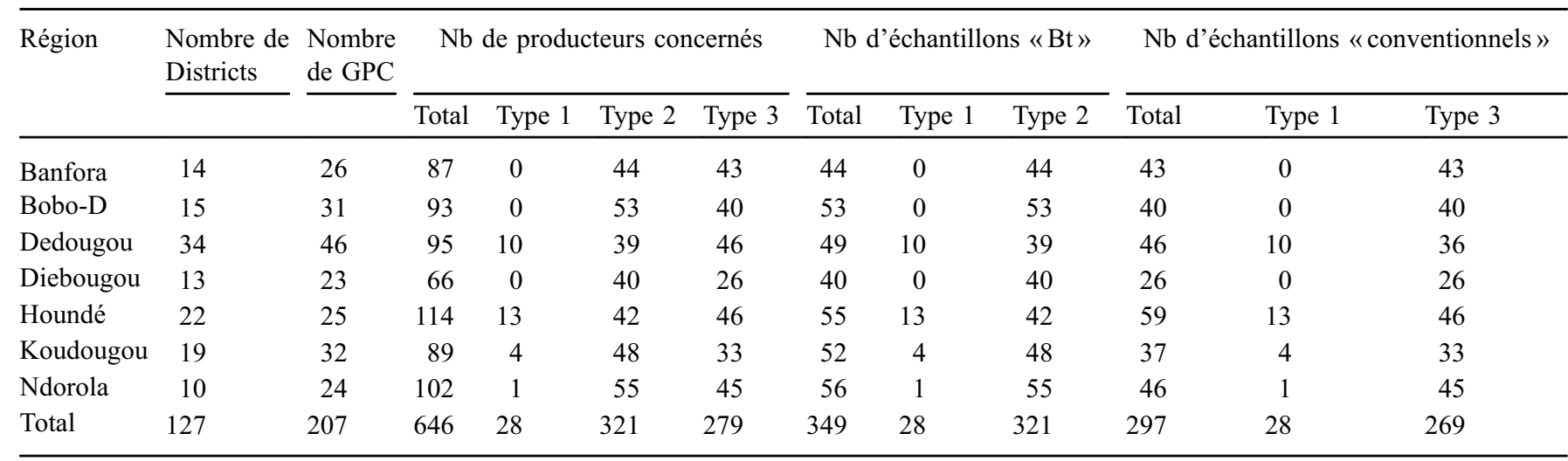

Type 1 = producteurs échantillonnés pour leurs parcelles Bt et conventionnelles ; Type 2 =producteurs échantillonnés pour leurs seules parcelles $\mathrm{Bt}$; Type 3 = producteurs échantillonnés pour leurs seules parcelles conventionnelles.

travers des lignes diagonales du champ. Trois capsules ont été prélevées au bas, au milieu et au sommet de chaque plante pour être égrenées. Selon les procédures suivies au laboratoire de contrôle de la qualité des graines (Sofitex, s.d.), onze graines ont été isolées au hasard pour subir un test ELISA et vérifier la présence ou non de Cry1Ac et de Cry2Ab. Pour chaque échantillon de champ, la présence des gènes Bt a été contrôlée pour chacune des onze graines, mais les résultats sont restitués de manière synthétique en indiquant les pourcentages de graines testées positives (par exemple, 9,09\% signifie qu'une graine a été testée positive sur les onze). Pour chaque échantillon, les résultats ont concerné la présence de CrylAc et/ou de Cry2Ab.

Pour statuer sur la nature, conventionnelle ou transgénique, des parcelles échantillonnées, nous avons tenu compte des modalités de la Sofitex pour les tests ELISA pour décider des seuils de présence des gènes $\mathrm{Bt}$ à retenir. Les tests ELISA sur onze graines par échantillon sont réalisés sans répétition, selon une procédure recommandée par Monsanto et que ce dernier doit appliquer dans le contrôle des semences qu'il produit, 
Tableau 2. Présence de gènes Bt dans les graines issues des parcelles échantillonnées.

Table 2. Presence of Bt genes in seeds from sampled plots.

\begin{tabular}{|c|c|c|c|c|c|c|}
\hline & \multicolumn{3}{|c|}{$\begin{array}{c}\text { Gènes présents } \\
\text { dans échantillons de FK37 }\end{array}$} & \multicolumn{3}{|c|}{$\begin{array}{c}\text { Gènes présents } \\
\text { dans échantillons de FK95 BG II }\end{array}$} \\
\hline & Cry1Ac & Cry $2 \mathrm{Ab}$ & $\overline{\text { Cry } 1 \mathrm{Ac}+\text { Cry } 2 \mathrm{Ab}}$ & Cry1Ac & Cry2Ab & Cry1Ac + Cry $2 A b$ \\
\hline $\begin{array}{l}\text { Part des échantillons avec présence* } \\
\text { du gène Bt concerné, \% } \\
\text { Taux de présence du Cry1Ac, } \%\end{array}$ & 63,6 & 59,3 & 52,2 & 95,7 & 94,3 & 92,6 \\
\hline Écart type & 23,2 & & 22,5 & 11,9 & & 11,2 \\
\hline \multicolumn{7}{|l|}{ Taux de présence du Cry $2 \mathrm{Ab}, \%$} \\
\hline Moyenne & & 39,3 & 40,3 & & 86,5 & 86,6 \\
\hline Écart type & & 23,2 & 23,3 & & 12,2 & 12,1 \\
\hline
\end{tabular}

* La présence du gène Bt concerné est prononcée quand elle est détectée dans au moins une graine sur les onze de chaque échantillon de FK37 (coton conventionnel) et dans au moins 6 graines dans chaque échantillon de FK95 BG II.

Tableau 3. État de contamination des semences conventionnelles dans les échantillons prélevés dans les champs de coton conventionnel (\% des 297 échantillons).

Table 3. Contamination status of conventional seeds in samples from conventional cotton fields (\% of 297 samples).

\begin{tabular}{llcr}
\hline Contamination $^{*}$ par Cry1Ac & \multicolumn{2}{c}{ Contamination ${ }^{*}$ par Cry2Ab } & \multicolumn{2}{c}{ Oui } \\
\cline { 2 - 4 } & Non & $7,1 \%$ & $36,4 \%$ \\
Non & $29,3 \%$ & $52,2 \%$ & $63,6 \%$ \\
Oui & $11,4 \%$ & $59,3 \%$ & $100,0 \%$ \\
Total & $40,7 \%$ & $0.3 \%$ & \\
\hline
\end{tabular}

* La contamination est prononcée quand la présence du gène Bt concerné est observée dans au moins une graine testée sur les onze de chaque échantillon.

mais sans jamais partager les résultats pour permettre une appréciation externe. La procédure suivie devrait être correcte pour l'échelle de production des grands agriculteurs des ÉtatsUnis, mais pas forcément valable pour celle des paysans cotonniers en Afrique. La variabilité du prélèvement des onze graines sur un échantillon peut être beaucoup plus forte au Burkina Faso. Il ne nous paraît donc pas raisonnable d'appliquer les valeurs habituelles de $0,5 \%$ et de $98,0 \%$ comme seuil maximal et seuil minimal pour nous prononcer respectivement sur la nature conventionnelle et transgénique des parcelles, car ces valeurs nous conduiraient immanquablement à conclure que les parcelles n'avaient pas la nature qu'elles devaient avoir. Aussi, nous avons retenu des seuils nettement moins contraignants, soit un seuil de $10 \%$ de présence maximale et un seuil de $55 \%$ de présence minimale pour indiquer respectivement la nature conventionnelle et transgénique des parcelles. Ces seuils, retenus de manière arbitraire mais pour éviter une exigence déraisonnable, correspondent respectivement à un résultat positif sur une graine et sur six graines sur onze.

Une parcelle pouvait être considérée comme de nature transgénique sans que nous puissions affirmer que les semences utilisées pour l'installer avaient le niveau désiré de pureté selon le taux de présence de gènes Bt. Il s'agit d'une question rarement abordée car on suppose que les semences génétiquement modifiées fournies sont nécessairement pures. Pour nous prononcer, nous avons retenu le seuil minimal de présence des gènes $\mathrm{Bt}$ de $90 \%$ (soit présence attestée dans au moins 10 graines sur 11) pour supposer que les semences ayant permis le semis du champ étaient effectivement de nature $\mathrm{Bt}$ suffisante. Il s'agit encore d'un seuil arbitraire, retenu pour éviter une exigence déraisonnable.

\section{Résultats}

Pour les échantillons issus de parcelles de nature supposée conventionnelle, la présence de gènes $\mathrm{Bt}$ a été clairement révélée dans les graines issues de ces champs (Tab. 2, colonnes 2 à 4). Sur les 297 parcelles concernées, 63,6\% et 59,3\% ont respectivement montré la présence de Cry1AC et de Cry2Ab. Dans les deux cas, le niveau de présence était élevé (plus de $40 \%$, ce qui correspond à un test positif observé pour 4 à 5 graines sur 11).

La nature supposée conventionnelle des parcelles était confirmée seulement pour 29,3\% d'entre elles, où aucun des deux gènes Bt n'a été détecté. Pis, la présence des deux gènes Bt a été constatée dans 52,2\% de ces parcelles, qui étaient non seulement de nature transgénique mais de nature Bollgard II 
Tableau 4. Taux de présence des gènes Bt dans les parcelles supposées transgéniques répondant au critère retenu pour juger de l'utilisation de semences suffisamment pures.

Table 4. Presence rates of Bt genes in supposedly transgenic plots meeting the criterion of purity of the seeds used.

\begin{tabular}{|c|c|c|c|}
\hline & Cry1Ac & Cry2Ab & Cry $1 \mathrm{Ac}+$ Cry2Ab \\
\hline $\begin{array}{l}\text { \% d'échantillons correspondant aux champs cultivés } \\
\text { à partir de semences pures }{ }^{*} \text { selon le gène Cry } \\
\text { Taux }{ }^{* *} \text { de présence de Cry } 1 \text { Ac dans les échantillons } \\
\text { des champs à partir de semences pures pour ce trait, \% }\end{array}$ & 59,6 & 53,6 & 40,4 \\
\hline Moyenne & 96,0 & & 96,7 \\
\hline \multicolumn{4}{|l|}{$\begin{array}{l}\text { Taux }{ }^{* *} \text { de présence de Cry } 2 \mathrm{Ab} \text { dans les échantillons } \\
\text { des champs à partir de semences pures pour ce trait, \% }\end{array}$} \\
\hline Moyenne & & 95,4 & 95,9 \\
\hline Écart type & & 4,6 & 4,5 \\
\hline
\end{tabular}

* Le champ est considéré avoir été semé avec des semences pures pour le trait Bt considéré si ce trait est détecté dans au moins 10 graines sur les 11 prélevées sur l'échantillon du champ.

** Taux calculé à partir du nombre de graines à présence détectée du gène Cry sur les onze de chaque échantillon.

Tableau 5. Statut réel des parcelles échantillonnées supposées transgéniques selon le critère retenu de pureté des semences Bt. Table 5. Real status of supposedly transgenic plots according to the purity criterion of the seeds used.

\begin{tabular}{llrr}
\hline Statut ${ }^{*}$ Cry1Ac & \multicolumn{3}{c}{ Statut ${ }^{*}$ Cry2Ab } \\
\cline { 2 - 3 } & Non & Oui & \multirow{2}{*}{ Total } \\
\hline Non & $27,2 \%$ & $13,2 \%$ & $40,4 \%$ \\
Oui & $19,2 \%$ & $40,4 \%$ & $59,6 \%$ \\
Total & $46,4 \%$ & $53,6 \%$ & $100,0 \%$ \\
\hline
\end{tabular}

* Le statut se réfère à l'utilisation effective de semences pures, c'est-à-dire une présence de gène Bt dans au moins $90 \%$ des graines testées à partir des échantillons, ou une présence dans au moins 10 graines sur les 11 prélevées dans chaque échantillon.

(Tab. 3). Il y avait $11,4 \%$ et $7,1 \%$ des parcelles qui étaient transgéniques respectivement par la présence du seul gène CrylAc et du seul gène Cry2Ab.

La nature supposée transgénique des parcelles était moins discutable, quoiqu'imparfaite. Parmi les 349 parcelles concernées, environ $95 \%$ ont dépassé le seuil retenu de présence de gène $\mathrm{Bt}$ pour chacun des deux gènes $\mathrm{Bt}$ considéré séparément (Tab. 2, colonnes 5 à 7). Même lorsque les deux gènes $\mathrm{Bt}$ étaient considérés conjointement, le seuil retenu a été dépassé dans $92,6 \%$ des parcelles concernées. Le niveau de présence du gène Bt était élevé, dans plus de $86 \%$ des graines testées dans tous les cas (soit plus de 9 graines sur 11).

Cependant, il serait plus difficile d'affirmer que les parcelles de coton Bt avaient été semées avec des semences ayant le niveau de pureté souhaitable au regard de la présence des gènes $\mathrm{Bt}$ (Tab. 4). Le critère de pureté des semences a été rempli pour moins de $60 \%$ des parcelles lorsque les deux gènes $\mathrm{Bt}$ ont été considérés séparément (Tab. 4), pour $40 \%$ seulement des parcelles lorsque les deux gènes étaient pris conjointement. Pour ces $40 \%$ de parcelles, les gènes $\mathrm{Bt}$ (Cry1Ac ou Cry2Ab) étaient présents dans plus de $95 \%$ des graines testées.

Si l'on considère le critère de pureté des semences pour définir la nature réelle du coton cultivé (conventionnelle ou $\mathrm{Bt}$ ) dans les parcelles supposées transgéniques, 27,2\% de ces parcelles étaient en fait de statut conventionnel (Tab. 5). Seules $40,4 \%$ des parcelles avaient effectivement la nature supposée de BG II (avec 2 gènes Bt). Pour le reste, 19,2\% des parcelles étaient de nature BG I (avec le seul gène Cry 1Ac) et 13,2\% des parcelles comportaient seulement le gène Cry2 Ab, type de coton Bt qui n'a jamais été commercialisé dans le monde.

\section{Discussion}

Notre étude visait à cerner la pureté des semences, au regard de la présence de gènes $\mathrm{Bt}$, utilisées pour les parcelles en coton conventionnel et transgénique. Nos résultats montrent que peu de parcelles avaient la nature supposée conventionnelle selon les informations obtenues de la Sofitex. Les parcelles supposées transgéniques l'étaient bien, mais souvent sans contenir les deux gènes $\mathrm{Bt}$ attendus.

Les distorsions constatées dans la nature des parcelles supposées conventionnelles sont grandes, mais elles peuvent découler en partie des pratiques évoquées d'arrangements entre les paysans, à l'insu de la Sofitex. Les valeurs des moyennes des taux de présence des gènes relatives à ces parcelles, moitié moindres que celles des parcelles de nature 
transgénique (Tab. 2), indiquent qu'elles n'avaient pas été installées entièrement avec des semences de nature transgénique. Néanmoins, la présence des deux gènes $\mathrm{Bt}$ dans $52,2 \%$ de ces parcelles indique qu'une part importante d'entreelles n'avaient pas été installées avec les semences conventionnelles que la Sofitex avait fournies. Ce résultat témoigne d'un manque de maîtrise des types de semences utilisées au niveau des producteurs qui, s'il concerne aussi les producteurs impliqués dans la production de semences de coton conventionnel, ne peut qu'induire un manque de pureté des semences obtenues.

Nos résultats de manque de pureté des semences transgéniques sont en accord avec le peu de littérature à ce propos. En Chine, l'analyse des teneurs en toxines Bt dans les plants a amené à soupçonner le manque de pureté des semences des variétés de coton Bt dans la province du Shandong (Pemsl et al., 2005) et dans les provinces de la vallée du Yangtsé où les variétés hybrides étaient utilisées (Xu et al., 2008). Dans les deux cas, l'ampleur de l'imperfection de la pureté n'a pas été estimée, contrairement au Pakistan. Dans ce dernier pays, où un seul gène Bt était utilisé (Cry1Ac), il a été constaté que $11 \%$ des agriculteurs croyaient cultiver du coton Bt alors que le gène Bt n'était pas présent, et a contrario $5 \%$ des agriculteurs croyaient cultiver du coton conventionnel alors que le gène Bt était présent (Spielman et al., 2017). Les valeurs plus élevées de divergence trouvées dans notre étude peuvent découler de l'utilisation combinée de deux gènes Bt au Burkina Faso.

$\mathrm{Au}$ Burkina Faso, les références sur la vraie nature des parcelles en coton Bt sont rares. Nous ne pouvons nous référer aux résultats des tests que la Sofitex réalise, mais ne partage pas. Dans une étude ayant permis de conduire une petite action pour vérifier le statut $\mathrm{Bt}$ des plants de coton dans les champs supposés être semés en coton Bt au Burkina Faso, Fok et al. (2016) ont observé que dans les tests effectués sur 45 échantillons, aucun gène $\mathrm{Bt}$ n'était présent dans $24,4 \%$ des échantillons, un seul gène Bt a été détecté dans 17,8\% des échantillons (fréquence également répartie entre chacun des deux gènes $\mathrm{Bt}$ ), et les deux gènes n'ont été trouvés que dans $57,8 \%$ des échantillons. Ces chiffres sont cohérents avec ceux de la présente étude qui est cependant basée sur un nombre plus élevé d'échantillons.

Davantage de travaux ont traité du phénomène de la contamination des semences conventionnelles par les gènes $\mathrm{Bt}$, mais avec beaucoup moins d'évaluation quantitative que dans notre étude. En plus des États-Unis évoqués en introduction, Kranthi et al. (2017) ont indiqué qu'en Inde, près de $30 \%$ des semences conventionnelles fournies pour le semis des parcelles refuge (des ravageurs cibles des gènes $\mathrm{Bt}$ selon la technique recommandée pour prévenir leur résistance aux gènes utilisés) contenaient des gènes Bt. Au Burkina Faso, $50 \%$ des producteurs de coton biologique ont reçu des semences contenant des gènes Bt (Vognan et Bourgou, 2014).

Nos résultats montrent clairement que, à la veille de la suspension de l'utilisation du coton Bt, les semences conventionnelles n'étaient pas pures et étaient largement contaminées. À l'opposé de l'étude du Pakistan citée, où la source des semences utilisées par les paysans n'a pas été contrôlée et pouvait difficilement l'être dans un contexte d'acquisition des semences au marché, on peut affirmer que le manque de pureté des semences conventionnelles observé au
Burkina Faso découlait pour beaucoup des défauts et distorsions que nous avons examinés dans la production, la distribution mais aussi l'utilisation des semences. Il en a résulté une défaillance, au sein de la Sofitex, dans le contrôle $\mathrm{du}$ respect des bonnes pratiques à toutes les étapes du processus, des champs jusqu'aux usines, alors que le contrôle était rendu davantage nécessaire par les pratiques opportunistes des paysans, que les changements dans les conditions de distribution des semences avaient par ailleurs favorisées. La défaillance mentionnée implique que la contamination des semences pouvait intervenir à n'importe quelle étape du processus et qu'elle était probablement intervenue à toutes les étapes de ce processus. La défaillance observée met aussi en exergue la dimension de coopération nécessaire entre les acteurs pour produire et préserver des semences de qualité.

Les caractéristiques du manque de pureté des semences transgéniques constaté ne permettent pas d'attribuer la responsabilité du manque seulement au système semencier du Burkina Faso et aux pratiques d'arrangements entre les paysans. La nature transgénique des semences est avérée, mais pas du tout pour ce qui concerne la nature BG II avec double présence des gènes $\mathrm{Bt}$ voulus. Comme nous pensons que cette double présence était déjà insuffisante dans les semences abusivement appelées G2 lors de leur livraison en juin 2008 par Monsanto, la responsabilité première de ce dernier est à souligner. Ensuite, il y a une responsabilité partagée entre Monsanto et le Burkina Faso pour ne pas avoir mis en œuvre des actions pour assurer, dès le départ, le meilleur taux de double présence des gènes $\mathrm{Bt}$.

Le défaut de pureté des semences des deux types de coton induit une première conséquence peu évoquée sur le contrôle des ravageurs cibles des gènes $\mathrm{Bt}$ et l'évaluation du gain potentiel apporté par le coton Bt. D'un côté, les champs supposés être en coton conventionnel étaient en réalité partiellement transgéniques et ils ont bénéficié partiellement de l'efficacité des gènes Bt contre les ravageurs ciblés. De l'autre, les champs supposés être transgéniques ne l'étaient pas parfaitement, de sorte que l'efficacité obtenue était inférieure à ce qu'elle aurait dû être. Il en découle un « effet ciseau» sur le gain potentiel, qui aurait dû être supérieur à celui mesuré par Vognan et Fok (2019). Selon l'évolution du gain de rendement constaté par l'Association interprofessionnelle du coton du Burkina (AICB, 2015) sur l'ensemble de la production nationale (donc peu sensible aux erreurs d'estimation à l'échelle d'un producteur), le gain apporté par le coton Bt a été de $18 \%, 9 \%, 9 \%, 5 \%, 12 \%$ et $15 \%$ pour les années successives allant de 2009 à 2014. La baisse du gain à la deuxième, troisième et quatrième année d'utilisation du coton $\mathrm{Bt}$ tend à confirmer l'hypothèse émise. Il reste que le gain s'est redressé par la suite, mais cela peut être la conséquence d'un meilleur respect des traitements de fin de cycle que certains paysans rechignaient à faire au départ (Vognan et Fok, 2019).

Le défaut de pureté des semences Bt, et plus particulièrement de la nature BG II, est néfaste pour l'efficacité et la durabilité de son utilisation contre les parasites ciblés. Les semences utilisées au Burkina Faso correspondaient en fait à un mélange à l'emballage des semences, dont le manque d'efficacité a été prouvé. Sun et al. (2013) ont montré qu'un mélange de 20 à $50 \%$ de semences conventionnelles à l'emballage conduisait à une efficacité faible à très faible de la résistance aux ravageurs ; or c'est ce niveau qui a été observé 
dans notre étude. Pis, il s'agit d'un type de mélange plus complexe, avec des graines Bt monogénique, dont l'utilisation a été la source d'apparition rapide de la résistance du ver rose en Inde (Tabashnik et Carrière, 2010). On pourrait arguer que le Burkina Faso pratiquait, sans le savoir, une démarche de refuge dans la parcelle (ou "refuge dans le sac») dont Tabashnik (1994) avait néanmoins signalé la faible efficacité, alors que la sélection de ravageurs à résistance dominante aux gènes Bt était favorisée (Brévault et al., 2015). Il est fort à craindre que, du fait du défaut de pureté des semences de coton Bt dès leur utilisation à grande échelle en 2008, la sélection d'individus résistants à chacun des deux gènes Bt a déjà eu lieu, facilitant ainsi la sélection d'individus résistants aux deux gènes. Il se peut même que la présence de ces derniers soit déjà effective. Si tel est le cas, l'intérêt de reprendre l'utilisation du coton Bt BG II avec les deux gènes déjà utilisés est fortement réduit.

\section{Conclusion}

À la veille de sa suspension de l'utilisation du coton Bt, le Burkina Faso n'avait pas échappé au phénomène de perte de pureté des semences conventionnelles, fortement contaminées par les gènes $\mathrm{Bt}$, alors que le taux de présence des deux gènes Bt dans les semences transgéniques était de $40 \%$ seulement. Les défauts observés pour les deux types de semences ont découlé d'une part de défaillances dans le contrôle des bonnes pratiques à toutes les étapes du processus de production de semences, alors même que les pratiques des paysans pour acquérir des semences supplémentaires auraient dû rendre ce contrôle plus rigoureux, et d'autre part de l'insuffisante qualité des semences fournies par Monsanto qui ne contenaient pas systématiquement les deux gènes Bt. L'utilisation de telles semences transgéniques imparfaites, dès le départ, a nui à l'efficacité de leur utilisation et a probablement déjà hypothéqué la durabilité de cette utilisation par la sélection de ravageurs résistants aux gènes utilisés.

Le cas étudié souligne l'importance d'un bon fonctionnement du système semencier, qui assure et préserve la pureté des semences produites, pour l'efficacité et la durabilité de l'utilisation des semences transgéniques. Il révèle aussi, indépendamment de l'utilisation de ces semences, le besoin d'ajuster le système de production de semences cotonnières en rapport avec les niveaux de production cotonnière atteints en Afrique francophone et les limites d'un système internalisé basé sur des agents de terrain multitâches. La rédaction d'un document de procédures nationales, comme le Burkina Faso s'y est déjà engagé, est une démarche nécessaire. Mais elle ne sera efficace que, d'une part, si les procédures tiennent compte d'une distribution réaliste des responsabilités et des moyens entre les acteurs impliqués, et d'autre part, s'il y a un esprit de coopération pour que la qualité des semences, et plus particulièrement la pureté génétique, soit perçue comme l'affaire de tous, y compris des paysans utilisateurs.

Remerciements. Cet article est une version française substantiellement remaniée d'un article précédemment publié en anglais sous licence CC-BY: Bourgou L, Kargougou E, Sawadogo M, Fok M. 2020. Bt cotton seed purity in Burkina
Faso: status and lessons learnt. J Cotton Res 3: 30. https://doi. org/10.1186/s42397-020-00070-4.

\section{Références}

AICB. 2015. Memorandum sur la production et la commercialisation du coton génétiquement modifié au Burkina Faso. Ouagadougou (Burkina Faso): Association interprofessionnelle du coton du Burkina (AICB).

Bourgou L, Hema SO, Koulibaly B, Diane SK, Sawadogo M. 2020. Impact of transgenic conversion on the characteristics of Burkina Faso cotton. African Journal of Biotechnology 19(1): 8-17. DOI: 10.5897/AJB2019.16907.

Brévault T, Tabashnik BE, Carrière Y. 2015. A seed mixture increases dominance of resistance to Bt cotton in Helicoverpa Zea. Scientific Reports 5: 1-7. DOI: 10.1038/srep09807.

Cederholm H. 2014. Protecting organic seed integrity. The organic farmer's handbook to GE avoidance and testing. Bridgewater (USA). Organic Seed Grower and Trade Association (OSGATA), $70 \mathrm{p}$.

Fok M. 1993. Le développement du coton au Mali par analyse des contradictions : les acteurs et les crises de 1895 à 1993. Document de travail. Montpellier (France) : CIRAD, $263 \mathrm{p}$.

Fok M. 1995. Le coton en Afrique francophone: révolution verte ou évolution institutionnelle? In: M. Griffon (Ed.). Succès et limites des révolutions vertes. Montpellier (France) : CIRAD, pp. 80-92.

Fok M. 2016. Impacts du coton Bt sur les bilans financiers des sociétés cotonnières et des paysans au Burkina Faso. Cahiers Agricultures 25(3): 1-13. DOI: 10.1051/cagri/2016020.

Fok M, Xu N. 2010. Libéralisation et régulation des marchés de variétés et de semences : le cas du coton Bt en Chine et dans les pays émergents. Cahiers Agricultures 19(1): 28-33. DOI: 10.1684/ agr.2009.0356.

Fok M, Xu N. 2011. Variety market development: a Bt cotton cropping factor and constraint in China. AgBioForum 14(2): 47-60.

Fok M, Neirac C, Renou A, Dessauw D. 2016. Étude de faisabilité du coton génétiquement modifié en Côte d'Ivoire. Montpellier (France) : CIRAD.

Food Democracy Now!. 2014. Coexistence equals contamination: tell the USDA to stop Monsanto's GMO contamination. [June 25, 2016]. www.fooddemocracynow.org/blog/2014/mar/3/tell the US DA_2_stop_Monsantos_GMO_contamination.

Kranthi S, Satija U, Pusadkar P, Kumar R, Shastri CS, et al. 2017. Non-Bt seeds provided by seed companies in India - are they suitable as refuge for Bt-cotton? Current Science 112(10): 25-26.

Luna JK, Dowd-Uribe B. 2020. Knowledge politics and the Bt cotton success narrative in Burkina Faso. World Development 136: 105127. DOI: 10.1016/j.worlddev.2020.105127.

Pemsl D, Waibel H, Gutierrez AP. 2005. Why do some Bt-cotton farmers in China continue to use high levels of pesticides. International Journal of Agricultural Sustainability 3(1): 44-56. DOI: $10.1080 / 14735903.2005 .9684743$.

Rabault M. 1944. Le coton dans les colonies françaises. Le passé. In : U.C. de l'Empire Français (Ed.). Conférences sur le coton. Paris (France) : Union Cotonnière de 1'Empire Français, pp. 18-34.

Savadogo N. 2015. Processus de production de la semence de coton à la Sofitex: contribution au suivi de la traçabilité de la semence. Mémoire de Master, Université 2iE, Ouagadougou (Burkina Faso). documentation.2ie-edu.org/cdi2ie/opac_css/doc_num.php?explnu m_id=2673. 
Singh BP, Sandhu SS, Kalia VK, Gujar GT, Dhillon MK. 2016. Assessment of Bt trait purity in different generations of transgenic cottons. Indian Journal of Experimental Biology 54(4): 237-244.

Sofitex. (date non indiquée). Le processus de contrôle de qualité de la semence (Tests spécifiques).

Spielman DJ, Zaidi F, Zambrano P, Khan AA, Ali S, et al. 2017. What are farmers really planting? Measuring the presence and effectiveness of Bt cotton in Pakistan. PLoS ONE 12(5): 1-10. DOI: 10.1371 /journal.pone.0176592.

Sun G, Zhang R, Wang Y, Chen T, Zhou Z, Rui Z, et al. 2013. Influence of seed purity on insect resistance and yield in transgenic cotton. Current Biotechnology 5(1): 27-31.

Tabashnik BE. 1994. Delaying insect adaptation to transgenic plants: seed mixtures and refugia reconsidered. Proceedings of the Royal Society B 255: 7-12. DOI: 10.1098/rspb.1994.0002.
Tabashnik BE, Carrière Y. 2010. Field-evolved resistance to Bt cotton bollworm in the US and pink bollworm in India. Southwestern Entomologist 35(3): 417-424. DOI: 10.3958/059.035.0326.

USDA. 2015. Coexistence fact sheets cotton. [ 12/06/2017]. http:// www.usda.gov/documents/coexistence-cotton-factsheet.pdf.

Vognan G, Bourgou L. 2014. Rapport d'étude sur la contamination du coton biologique par le cotonnier génétiquement modifié (CGM). Bobo-Dioulasso (Burkina Faso) : INERA, Programme coton, 63 p.

Vognan G, Fok M. 2019. Performance différenciée du coton Bt en début de diffusion: cas du Burkina Faso. Cahiers Agricultures 28: 26. DOI: $10.1051 /$ cagri/2019026.

Xu N, Fok M, Bai L, Zhou Z. 2008. Effectiveness and chemical pest control of Bt-cotton in the Yangtze River Valley, China. Crop Protection 27: 1269-76. DOI: 10.1016/j.cropro.2008.04.003.

Zangré GR. 2009. Du protocole de Cartagena à l'exploitation à grande échelle du coton Bt au Burkina Faso. Biotech Echo 27: 1-3.

Citation de l'article : Bourgou L, Fok M. 2021. Système semencier et pureté des semences dans un contexte d'utilisation d'OGM : le cas du coton Bt au Burkina Faso. Cah. Agric. 30: 25. 